\title{
Infrared Imaging of Boundary Layer Transition Flight Experiments
}

\author{
Scott A. Berry ${ }^{*}$ and Thomas J. Horvath, Jr. ${ }^{\dagger}$ \\ NASA Langley Research Center, Hampton, VA, 23681 \\ Richard Schwartz \\ ATK Space Division, Hampton, VA, 23681 \\ Martin Ross ${ }^{\S}$ \\ The Aerospace Corporation, Los Angeles, CA, 90009 \\ and \\ Brian Anderson $^{* *}$ and Charles H. Campbell ${ }^{\dagger \dagger}$ \\ NASA Johnson Space Center, City, Houston, TX, 77058
}

\begin{abstract}
The Hypersonic Thermodynamic Infrared Measurement (HYTHIRM) project is presently focused on near term support to the Shuttle program through the development of an infrared imaging capability of sufficient spatial and temporal resolution to augment existing on-board Orbiter instrumentation. Significant progress has been made with the identification and inventory of relevant existing optical imaging assets and the development, maturation, and validation of simulation and modeling tools for assessment and mission planning purposes, which were intended to lead to the best strategies and assets for successful acquisition of quantitative global surface temperature data on the Shuttle during entry. However, there are longer-term goals of providing global infrared imaging support to other flight projects as well. A status of HYTHIRM from the perspective of how two NASAsponsored boundary layer transition flight experiments could benefit by infrared measurements is provided. Those two flight projects are the Hypersonic Boundary layer Transition (HyBoLT) flight experiment and the Shuttle Boundary Layer Transition Flight Experiment (BLT FE), which are both intended for reducing uncertainties associated with the extrapolation of wind tunnel derived transition correlations for flight application. Thus, the criticality of obtaining high quality flight data along with the impact it would provide to the Shuttle program damage assessment process are discussed. Two recent wind tunnel efforts that were intended as risk mitigation in terms of quantifying the transition process and resulting turbulent wedge locations are briefly reviewed. Progress is being made towards finalizing an imaging strategy in support of the Shuttle BLT FE, however there are no plans currently to image HyBoLT.
\end{abstract}

\section{Introduction}

$\mathrm{H}^{5}$ ypersonic vehicles require a thermal protection system (TPS) for insulation against severe temperatures that result from the extreme velocities. Friction (or viscous dissipation) within the boundary layer is the underlying phenomenon for the temperature increase. Additionally, the aerothermal environment, which dictates the TPS design, will be made worse if the boundary layer around the vehicle becomes turbulent, as the friction-induced

\footnotetext{
* Aerospace Engineer, Aerothermodynamics Branch, M/S 408A, AIAA Senior Member.

$\dagger$ Aerospace Engineer, Aerothermodynamics Branch, M/S 408A, AIAA Associate Fellow.

\$Senior Research Scientist, support to Advanced Sensing and Optical Measurements Branch M/S 493, AIAA member.

${ }_{\text {Senior Research Staff Scientist, Space Launch Projects. }}$

** Orbiter Entry Aeroheating NASA Subsystem Engineer, Aeroscience and Applied CFD Branch, M/C EG3, AIAA member.

${ }^{\dagger}$ Aerospace Engineer, Applied Aeroscinces and CFD Branch, M/C EG3, AIAA Senior Member.
} 
temperatures will be forced closer to the surface. Thus the accurate prediction of boundary layer transition (BLT) onset allows for better-defined aerothermal environments and then, potentially, TPS designs with less conservatism. Unfortunately, an incomplete physical model of the transition process presently impedes the development of highfidelity numerical tools for the reliable and rapid prediction of BLT on complex vehicle shapes.

During the recent return to flight (RTF) activity to resume Shuttle operations after the Columbia accident investigation, a BLT prediction tool based on an engineering level approach was developed (see Refs. 1 - 6) that is now part of the on-orbit (or mission) evaluation of Orbiter TPS health prior to entry. Launch-induced damage, in the form of small gouges (or cavities) to the ceramic tile surface or protrusions of the gap fillers (normally between the tiles), has the potential to reduce TPS margins (the built-in conservatism) below acceptable levels for safely withstanding the entry environment. The damage assessment process was established to better understand and monitor launch-induced damage from a mission and safety assurance perspective.

Presently there are two NASA-sponsored flight experiments with the goal of investigating hypersonic boundary layer transition. The Shuttle BLT flight experiment intends to conduct an Orbiter entry flight test to acquire hypersonic, high enthalpy aerothermodynamic environment and boundary layer transition data with a fixed protuberance. The fixed geometry is to be machined out of a monolithic BRI-18 tile, and located on the Orbiter wing in a location near the port wing landing gear door. The aerothermal technical community hopes to utilize a total of three flights to obtain Orbiter BLT flight data with increasing protuberance heights, however only one is currently approved. The three flights are intended to generate BLT onset data for free stream Mach numbers between 15 and 19. Discussions will continue between now and the completion of the first flight (currently scheduled for early 2009) on the technical and financial merits of these additional flights (see Ref. 7). The second flight test program, called HyBoLT (for hypersonic boundary layer transition), is expected to launch this summer (2008) to acquire flight BLT data with local conditions appropriate for the Shuttle during entry. Reference 8 provides an overview of this activity. Both of these flights are intended to help reduce uncertainties associated with the use of the BLT Tool outside of the range of flight conditions presently available from the historical calibration data.

The Hypersonic Thermodynamic Infrared Measurement (HYTHIRM) project sponsored by NASA's Engineering and Safety Center (NESC) is currently dedicated to obtaining global infrared images of the Shuttle during entry of sufficient spatial, thermal, and temporal resolution to augment the existing on-board instrumentation. Reference 9 provides an overview of this effort and is intended as part of a series of four papers to be presented together at the $40^{\text {th }}$ AIAA Thermophysics Conference in Seattle, WA on the HYTHIRM activity. An initial task within this effort was directed at defining Shuttle specific spatial and spectral sensor requirements to enable the successful acquisition of flight global surface temperature data. This led to new optical and radiance modeling tools for assessing requirements against existing imaging platforms within NASA, the Department of Defense, and the private sector. A systems trade study (see Ref. 10) was performed in order to optimize imaging strategies and provide recommendations regarding enabling technologies to meet Shuttle spatial and spectral requirements. Also, these new tools were validated during a semi-remote field deployment of several optical assets, at the Sandia National Laboratory National Solar Thermal Test Facility (NSTTF), whereby radiometric data was collected on a Shuttle tile array heated to entry-like surface temperatures. The objective of the NSTTF test (see Ref. 11) was to assess radiance and atmospheric modeling mission-planning tools and also to provide radiometric calibration of the imagers. Finally, the intent is to develop a forward plan to most effectively leverage this unique opportunity to obtain hypersonic flight boundary layer transition data using global infrared thermography. References 12 and 13 , both presented previously, provide additional details relative to the HYTHIRM activity. The present paper (the last of the four) is intended to provide an update on the status of these two NASA-sponsored flight projects and details about using available IR systems to image them.

\section{RTF BLT Tool V2 and correlations}

A program was established, in support of the Shuttle RTF effort, to develop a boundary layer transition predictive capability using ground-based empirical correlations. ${ }^{1}$ This effort resulted in a mission support tool that is integral to the damage assessment process for Orbiter TPS health monitoring, which has been used during every mission since the Columbia accident. The BLT Tool predicts transition onset time during entry for both protuberances and cavities, based on the ground-based developed correlations that were calibrated against a limited set of historical flight transition data. Recently, this tool has been updated to include additional experimental results, a new more in-depth review of the flight data, and a different computational approach, all of which were intended to reduce uncertainties with the final correlations. 
The initial version of the BLT tool was developed primarily with ground-based data (from perfect gas facilities with lower enthalpy and Mach numbers than flight) and as such required high quality flight calibration cases to ensure proper scaling of the empirical correlation to flight. The updated tool (V2) has tried to enhance the initial wind tunnel database by expanding the testing range to higher Mach and enthalpy conditions, but even still cannot match the conditions of flight. The current BLT Tool methodology has significant uncertainty with the assessment of transition onset predictions that are outside of the range of the existing flight calibration cases.

\section{A. Wind Tunnel to Flight Uncertainty}

An observation resulting from the recent in-depth review of the historical Shuttle flight transition data ${ }^{5}$ is that it is of limited range (transition at or below Mach 18) and less than optimum quality. The historical data initially set expectations of BLT no earlier than Mach 18. In reality, almost all of the missions since the tool was implemented have had to make mission support decisions even though the predicted BLT onset times have been much earlier (or higher Mach numbers) than the historical data, and thus outside our range of calibration and experience. Furthermore, of the flight cases in which there was sufficient documentation to be able to connect cause and effect between the offending trip and the measured transition onset time during flight, none of those cases had any information on the actual dimensions of the trip prior to (which is most importance to the damage assessment team or DAT process) or during the reentry (due to gap filler bending ${ }^{14}$ or cavity growth). There is a definite need to understand how hypersonic BLT behaves beyond the existing historical flight range (thus even higher Mach and enthalpy conditions), and to acquire flight data that includes measurements taken prior to entry (a well controlled or understood experiment).

\section{B. Flight BLT Review}

As noted earlier, an in-depth review of the historical Shuttle flight data was completed as an input to the new version of the BLT Tool, the results of which are captured in Ref. 5. The goal of that effort was to determine the highest quality set of flight calibration cases, whereby the clear connection could be made between the cause, with sufficient definition in documentation of the location and dimensions of the trip, and effect, the measured transition onset time downstream that cannot be associated with any other site. Out of that effort, only 7 flight cases were identified for protuberances (gap fillers) and 5 for cavities that were considered of sufficient quality to use for calibration of the new version of the BLT Tool. The dearth of calibration cases was a surprise to the team. Further, the realization that critical details would never be known, such as what these historical trip sites looked like in orbit and prior to entry, or how the damage geometry may have deformed due to aerothermal and shear loads, was recognized as a concern, which ultimately led to higher uncertainties with the final correlations. Thus, post RTF flights were anticipated to provide a wealth of BLT calibration data with critical details recorded at all stages of the mission.

Indeed, these recent flights have underscored the vulnerability of the TPS to launch induced damage, and the capacity for that system to sustain damage and still land safely. Unfortunately, these flights have not provided nearly enough quality BLT calibration cases for significantly reducing tool uncertainties. The primary reason for that is that the lack of surface instrumentation has left many gaps in surface coverage for determining the effect of many of the key damage sites and trips. The following is a brief review of some of the more interesting recent flights with significance to both BLT and IR imaging.

The initial flight after the RTF effort was STS-114, which was a very significant mission from many perspectives. First, it represented the initial application of the BLT Tool, and thus there was some uncertainty about how the tool would perform during the mission. Second, two large protruding gap fillers towards the forward region of the windward surface were identified early in the mission from the on-orbit imagery. The size and location of both of these protrusions was critical, with very early (or high Mach number) BLT predictions and positioning such that both wing leading edges would have likely experienced unacceptable surface temperatures. Because of the uncertainty associated with very early transition and the subsequent effect on the wing

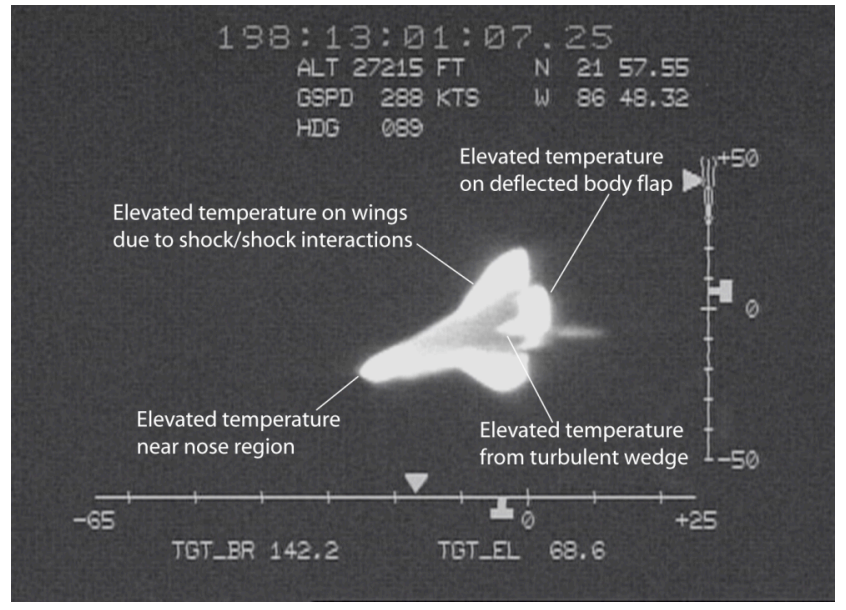

Figure 1. Initial unprocessed near infrared imagery from the Navy's Cast Glance aircraft during STS-121.

American Institute of Aeronautics and Astronautics 092407 
leading edge heating and structure, the decision was made by the mission management team (MMT) to remove these two gap fillers with an unprecedented spacewalking repair. Reference 6 provides additional details in regard to this mission and the resulting flight transition data that was acquired. Although early BLT onset time predictions were a concern during the mission assessment, once the two large gap fillers were removed the actual measured result was relatively nominal with transition onset just under Mach 8. Another interesting note for this mission was that it was the first attempt to acquire global infrared (IR) measurements during reentry using multiple airborne platforms (see Ref. 12). It turned out that due to inclement weather at the primary landing site at NASA Kennedy Space Center, the Orbiter was diverted to Edwards AFB. While no imagery data was collected with two NASA WB-57 aircraft and the Missile Defense Agency's Gulfstream (called HALO II), the logistics involved with communicating ground track updates and re-deploying the aircraft to support one orbit wave-offs was successfully demonstrated.

The next flight, STS-121, was also eventful with early BLT predictions, a decision to enter as-is, and the first successful acquisition of IR images showing a turbulent wedge at hypersonic conditions (see Fig. 1). Similar to the prior mission, there were two large gap fillers protrusions, except this time they were in the aft windward area. During the damage assessment for both of these sites, the local TPS and structure were cleared to handle the worst case predicted transition onset times and heating environments. Thus, the decision was made by the MMT to leave these gap fillers in place during entry. One was out on the port wing and fortunately there was a surface thermocouple directly downstream of it for establishment of the actual transition onset time for possible use as a calibration case. Based on the actual height as measured on the ground predicted transition onset was on the order of Mach 19, but was actually measured to be closer to Mach 17. The BLT tool prediction was conservative, but maybe overly so as this gap filler was nearly aligned with the local streamlines as opposed to being at a 45-deg angle to the flow like the vortex generators (diamond trips) that the wind tunnel database ${ }^{3}$ is based on. The RTF BLT Tool does not currently have any method with which to take into account the angle of the local streamlines in relation to the trip. The other gap filler was closer to centerline, just in front of the starboard external tank (ET) door, but did not have any thermocouples in the region for determination of the measured transition onset times. Again, three aircraft were utilized in another attempt to collect global imagery, this time with success. A Navy P-3 Orion (referred to as Cast Glance) was involved and deployed under the Orbiter ground track near a point in the planned trajectory where the Mach number was approximately 12. Two other aircraft (NASA's WB-57 and the HALO II), which had been used during the first attempt, were deployed at observation points closer to the nominal transition onset time of Mach 8. The Navy P3-Orion aircraft was able to capture approximately four minutes of imagery. Processed images from closest approach are shown in Fig. 2, both visible and near infrared (NIR), showing the imprint from a turbulent wedge near where the ET door gap filler would have predicted early transition, thus establishing that at least the ET door gap filler had promoted transition prior to Mach 12. The on-orbit prediction for this site was as early as Mach 23 based on the full height of the protrusion. However, due to the quality of the on-orbit photographs, the gap filler was identified to have a number of rips and creases, indicating that the gap filler would likely would bend over or rip away during entry. Transition onset prediction for this case was thought to be closer to Mach 18. Upon landing, the height of the ET door trip was confirmed to be the smaller height as large portions of the gap filler did in fact rip away during entry. Another piece of circumstantial evidence for early transition for this site was the pattern of discolored tiles identified on the runway that qualitatively matches with the turbulent wedge identified in the NIR image of Fig. 2. The port wing gap filler could not be seen in the images due to over saturation (the bright white regions) of the IR sensors from the higher temperatures on the wing. Reference 12 provides additional details about this mission and imaging results.

The next mission, STS-115, was somewhat less eventful, as it had been one of the cleanest of the first three since the Shuttle program was returned to flight. There were no major cavity sites and only one protrusion of concern. The location for this protrusion was in a similar position to the gap filler from the previous flight, except this time on the port side ET door, and was eventually determined to be a plastic shim stock that would quickly melt away during entry. The damage assessment process was completed quickly and the

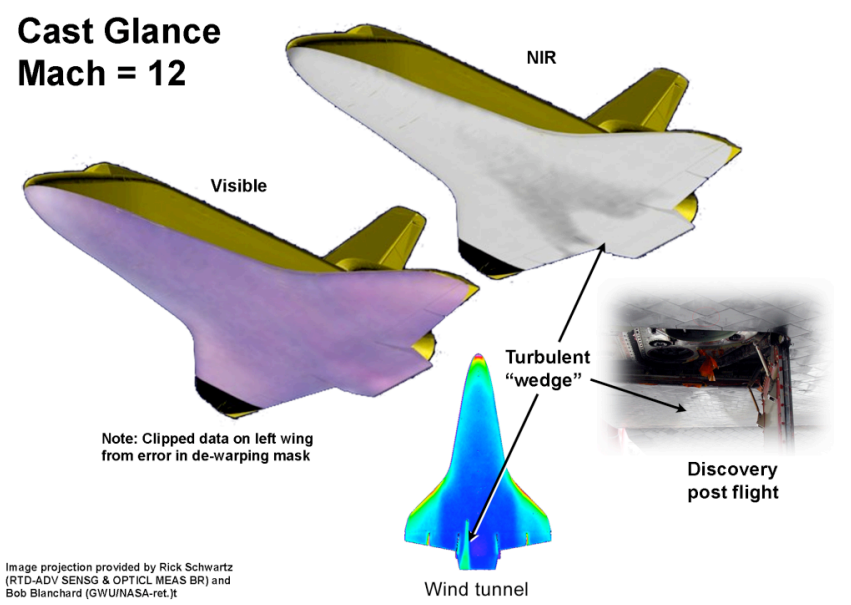

Figure 2. Comparison of various STS-121 images to wind tunnel results showing the turbulent wedge.

4

American Institute of Aeronautics and Astronautics 092407 
vehicle was cleared for reentry. The resulting measured transition onset was nominal, on the order of Mach 6 to 8, a clear indication of the lack of damage to the TPS for this flight. Entry imaging support to STS-115 provided two aircraft (one WB-57 and the Cast Glance). Cast Glance was stationed near the planned ground track at approximately Mach 14, as shown in Fig. 3, and visual and NIR imagery were collected. The multiple NIR images shown in Fig. 3, taken at different times along the trajectory, all show the improvement made between missions in reducing image saturation (the bright white regions). Reference 9 shows the final analyzed image, converted from intensity to temperature, with excellent comparison against the flight thermocouple data.

By comparing the two closest approach NIR intensity images in Figs. 2 and 3, the temperature augmentation just forward of the body flap for STS-

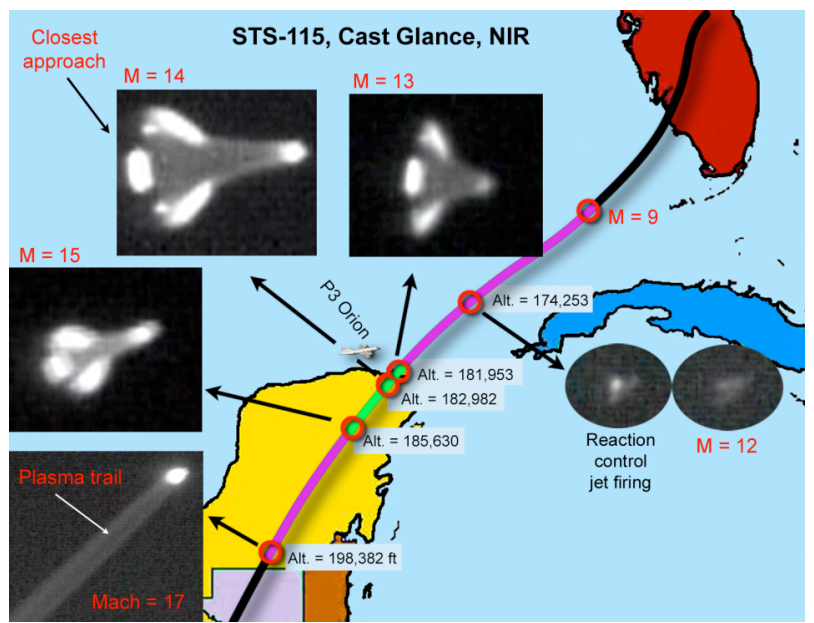

Figure 3. STS-115 near infrared imagery from the Navy's Cast Glance aircraft. 121, which is believed to be from the protruding gap filler, becomes more distinctive. Due to the lack of instrumentation in the aft region, these IR images have thus become invaluable for determining the presence of early BLT information that otherwise would have been lost for calibration purposes. However, in the case of the STS-121 gap filler, the onset information most critical to tool calibration was not picked up due to inadequate IR imaging coverage along the trajectory (i.e. temporal resolution).

While the next few flights were relatively quiet, STS-116 and STS-118 are both noteworthy primarily from a damage assessment team perspective. The STS-116 damage assessment process found little of concern during the mission, but upon entry was surprised by the somewhat early transition (Mach 13.5) picked up by the on-board instrumentation. A thermal barrier near the main landing gear door was found protruding on the runway and the measured height (0.2-in) and transition onset time matched well with the BLT Tool prediction. The size of this protrusion was just small enough to be missed by the on-orbit imagery used to identify damage sites. Luckily the on-board instrumentation was able to record this transition event (IR imaging aircraft were not available for this mission), as it currently is one of the best post-RTF calibration cases for the BLT Tool. On STS-118, there were 13 windward sites of interest during the mission, with one in particular providing the most concern. Shown in Fig. 4 is a photograph of a cavity that was identified from the launch and on-orbit imagery to be due to debris from the external tank that ricocheted off attachment hardware directly into the TPS, instead of being the usual glancing-type gouge. This deep cavity exposed the adhesive layer that bonds the tile to the sub-structure. The damage assessment process provided analysis that had many on the team concerned about whether this site would be allowed to reenter as is. In the end this site was cleared, but some on the team experienced some trepidation during entry. Post-flight inspection on the runway revealed no degradation of the damage site, as shown in Fig. 4 (a post-flight photo), in contradiction to the expectations of the team. Thus, the DAT tools over predicted the environments and thermal/structural responses of this site.

After the mission, there was an emphasis on trying to deconstruct the process to determine the source (or sources) of and quantify the level of the conservatism in this case. One obvious source was the BLT Tool, which is expected to set the proper aerothermal environments from which the thermal and structural analysis tools begin. Since the primary focus of almost all of the previous flights had been gap filler protrusions with very early transition times, the BLT Tool had never been truly tested against a cavity. The implementation of the BLT and cavity heating tools together ${ }^{2}$ required an untested assumption that, for lack of any defining data to the contrary, applied the predicted transition onset times instantaneously within the cavity. This assumption was based on the

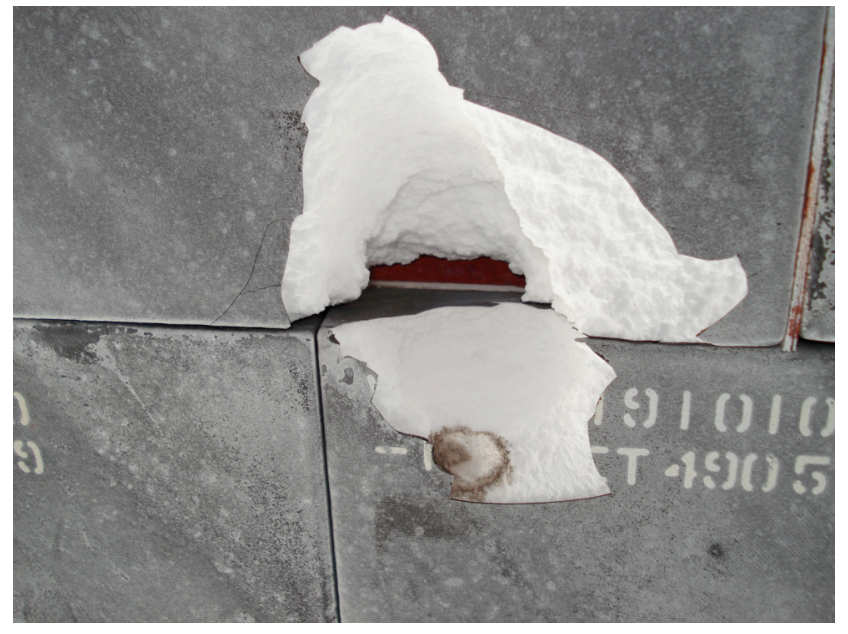

Figure 4. Cavity identified during STS-118. 
observation that in the wind tunnel cavity induced transition tended to jump abruptly to the site. The BLT Tool predicted transition onset for this deep cavity at around Mach 16. Applying turbulence within the cavity at this Mach number produced predictions of the thermal and structural response of the TPS that were inconsistent with the post-flight observations. Unfortunately in this case, the location of this damage site was such that there were not any surface thermocouples in the vicinity or downstream, and thus no post-flight data to accurately assess the best approach for applying transition to the cavity. Also, unfortunately, there were no airborne IR systems utilized for this flight to supplement the existing instrumentation and fill in the detail between sensors.

Thus, there have been at least two recent flights in which IR systems would have been the only way to acquire valuable flight transition data to help reduce BLT correlation uncertainties. One flight, STS-121, had an airborne IR platform with a qualified success of an observed turbulent wedge at Mach 12, but not sufficient coverage to see when BLT onset occurred (or when the gap filler was torn away). The other, STS-118, did not have IR data available and our ability to reconstruct the DAT process accurately, using mission and post-flight measurements and observations, for reduction of excess conservatism was severely hampered.

\section{Proposed Flight Experiments}

There are currently two NASA flight experiments that are under consideration to support the RTF BLT Tool wind tunnel to flight uncertainty assessment: HyBoLT and the Shuttle BLT FE. HyBoLT came about rather serendipitously as a flight of opportunity, when a previous flight test program was cancelled and the maiden launch of the ATK ALV X-1 rocket required a new first-stage payload. The BLT FE required a little more effort to gain momentum towards approval, utilizing a "white" paper to build advocacy for utilizing the Shuttle as a flight test vehicle. Both were proposed as a result of the experience during STS-114, whereby the need to understand and potentially minimize uncertainties to avoid an unnecessary EVA in the future was identified.

\section{A. HyBoLT}

A flight of opportunity was provided through the termination of a scramjet-powered test vehicle that was to be the primary payload on the maiden launch by ATK of a multi-stage rocket, the ALV X-1. The original agreement was to carry the Scramjet Flight Experiment (SFX) in exchange for NASA providing range support and launchindemnification. NASA terminated the SFX program in March 2006 due to cost and schedule concerns, which left the ALV X-1 without a primary payload for the top of the rocket. The HyBoLT (Hypersonic Boundary Layer Transition) flight experiment was then developed as a replacement for SFX with the goal of having minimal impact to the ALV X-1 launch schedule. The ALV X-1 is currently scheduled to launch from NASA Wallops Flight Facility (WFF) on the Northeastern shore of Virginia in the summer of 2008. Figure 5 provides an artistic rendering of HyBoLT and ALV X-1 launching from the WFF facility. The HyBoLT flight experiment was proposed to obtain code validation data for prediction of hypersonic boundary layer transition. The flight experiment actually has two sides with independent objectives, with one side dedicated to investigating smooth wall transition (referred to as Side A) and the other side dedicated to discrete roughness transition (Side B). The Side A experiment is designed to look at cross-flow transition at a Mach number near 3.5, acquiring data for verification of the physics-based model used to design the experiment. The Side B experiment was specifically proposed to provide flight BLT calibration data that represented a controlled experiment and at least partially simulated the proper local conditions at the trips to compare against the ground-based correlations. There are three trips on Side B, a cavity on centerline and two protrusions (a fence representing flight versus a diamond representing the wind tunnel) on either side, 5 -in off centerline. See Ref. 8 for additional details. A recognized shortcoming of the HyBoLT Side B experiment was that the freestream Mach and enthalpy conditions would not match flight, as the trips were sized to expect BLT onset at or around Mach 7, however the local conditions at the trips are appropriate for flight.

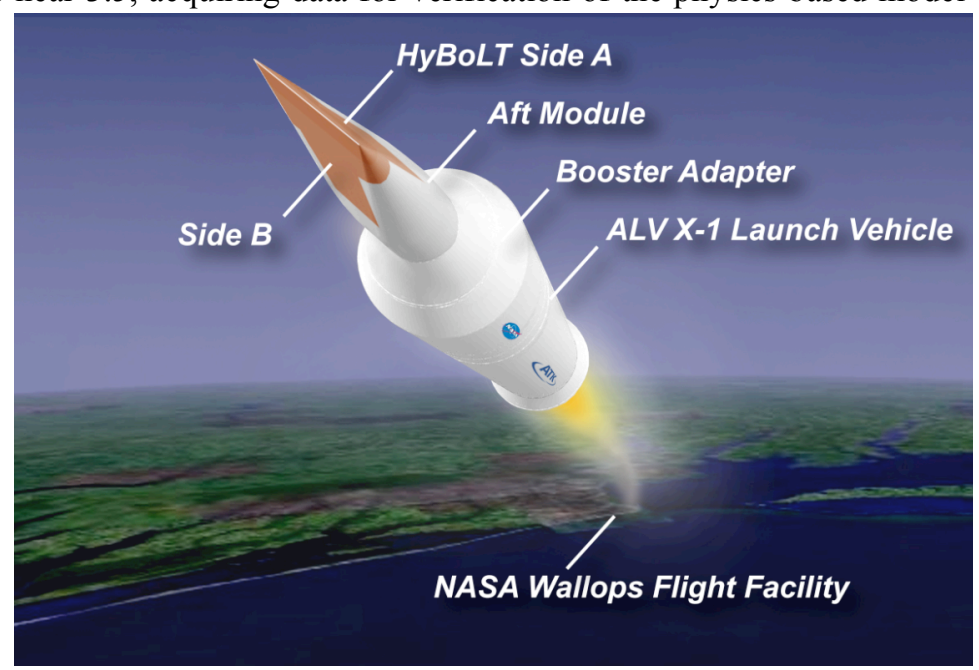

Figure 5. Artistic rendering of HyBoLT during launch from Wallops Flight Facility.

6

American Institute of Aeronautics and Astronautics 092407 


\section{B. Shuttle BLT Flight Experiment}

Boundary layer transition during hypersonic entry is one of several fundamental aerothermodynamic effects that influence the design and operation of manned spacecraft. Flight data acquired during the history of the Space Shuttle Program has provided important information regarding boundary layer transition onset and turbulent heating levels. However, the existing reentry boundary layer transition data on the Orbiter have been obtained as a result of uncontrolled changes in vehicle configuration mainly due to protruding gap fillers. Efforts to develop an approach for performing entry boundary layer transition flight-testing have led to the formation of a project activity intent on acquiring hypersonic flight data utilizing the Orbiter as a test vehicle. A preliminary proposal was developed to perform a variety of Orbiter entry flight experiments, which ultimately led to the decision to fund only the BLT FE. Current plans have identified a sequence of Orbiter boundary layer transition flight tests that could be accomplished before the end of Shuttle program in 2010, with a first flight planned for the early 2009 with STS-119. The BLT FE is based on using fixed protuberance geometry machined out of a monolithic BRI-18 tile, and is located near the port wing landing gear door (see Fig. 6). Also shown in the figure is the expected turbulent wedge, based on a nominal spreading angle of 15-deg, for a Mach 18 flight prediction. However, as of this moment, the Shuttle program has currently only approved the first flight. The technical community hopes for a total of three flights to obtain Orbiter flight data with increasing protuberance heights to generate BLT onset results for free stream Mach numbers between 15 and 19. While the first flight uses a trip height expected to induce BLT at Mach 15 to qualify the TPS margins, the last flight is required to extend beyond the range of the current historical data. Discussions will continue between now and the completion of the first flight on the technical and financial merits of the additional flights. Reference 7 provides details about plans for additional surface instrumentation dedicated for this experiment.

\section{Wind Tunnel Studies}

Wind tunnel entries into the Langley Research Center 20-In Mach 6 Tunnel ${ }^{15}$ were conducted for both the HyBoLT and Shuttle BLT FE projects, as part of a risk mitigation activity to better understand the transition process and resulting heating behind each trip. In particular, these test were conducted to quantify the movement of transition relative to each flight vehicle's thermocouple locations. Thus, turbulent wedge patterns were obtained for each configuration mainly as a function of Reynolds number and model orientation (angle of attack and yaw variations).

\section{A. HyBoLT Test}

Phosphor thermography and oil-flow data were acquired in the LaRC 20-In Mach 6 Tunnel during Test 6940 in July/August of 2007 to investigate streamlines and turbulent spreading as a function of off-nominal vehicle orientation ( $\alpha$ and $\beta$ of \pm 2 deg). In addition, a limited number of runs were dedicated to obtaining trip effectiveness data, for comparison against the Shuttle correlations that were used to size the trips for the flight vehicle.

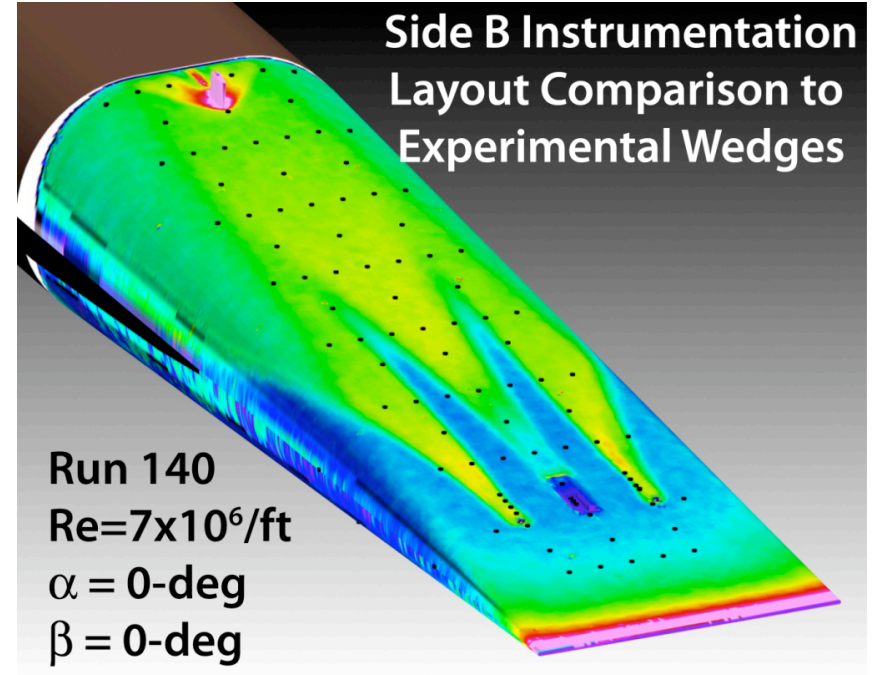

Figure 7. Wind tunnel results showing location of turbulent wedges in comparison to the HyBoLT flight vehicle instrumentation

7

American Institute of Aeronautics and Astronautics 
Also, there were runs without trips that were intended to provide natural transition patterns of interest for the Side A experiment, albeit at a freestream Mach number that was a bit higher (Mach 6) than when transition is expected to happen for Side A (Mach 3.5). An issue identified during this test was that the turbulent wedges developed from the two outboard trips were swept further outboard than expected. Figure 7 provides a sample result from one of the nominal Side B cases, in which the phosphor image is mapped to a three dimensional CAD file of the HyBoLT vehicle for comparison of the turbulent wedge patterns to the sensor layout. The sensor pattern is shown to be sufficient to pick up all three turbulent footprints when the trips are fully effective. One concern is that later in the trajectory, when the two outboard trips become less effective, or are just incipient, the disturbed flow will be swept towards the shoulder region in which there is no surface instrumentation. Figure 8 provides the effect of

Reynolds number, showing the location of the turbulent wedges for laminar, incipient and, effective conditions. Note that the size of the cavity and protuberances were approximately scaled such that they would trip within the Reynolds number range of the tunnel, and as a result the two protuberances are slightly more effective than the cavity. In flight, the three trips are expected to lose effectiveness at about the same point in the trajectory.

\section{B. Shuttle BLT FE Test}

Phosphor thermography data were acquired in the LaRC 20-In Mach 6 Tunnel during Test 6949 in May 2008 to investigate streamline locations and turbulent spreading as a function of off-nominal vehicle orientation ( $\alpha$ between 30 and $40 \mathrm{deg}$ and $\beta$ between $\pm 4-\mathrm{deg}$ ). In addition, a limited number of runs were dedicated to obtaining trip effectiveness data for comparison to the existing Shuttle correlations. Also, there were a few runs that investigated the effect of trip orientation and location. This test identified that the turbulent wedge in this case, as shown in Fig. 9, was slightly smaller and more inboard than expected based on the streamline predictions from flight conditions. Comparing Figs. 6 and 9 illustrates the smaller and more inboard turbulent footprint (note the wedge in reference to the elevon gap region). Further analysis is underway to try to understand if this will have any implications for the placement of the surface thermocouples for the flight experiment. Another observation that was surprising was that the location of the turbulent wedge seemed less sensitive to vehicle orientation effects. In other words, small angle of attack and yaw changes did not seem to appreciably affect the location of the wedge. For instance, Fig. 10 provides the effect of model yaw on the turbulent wedges emanating from the trip location on the wing. Note that
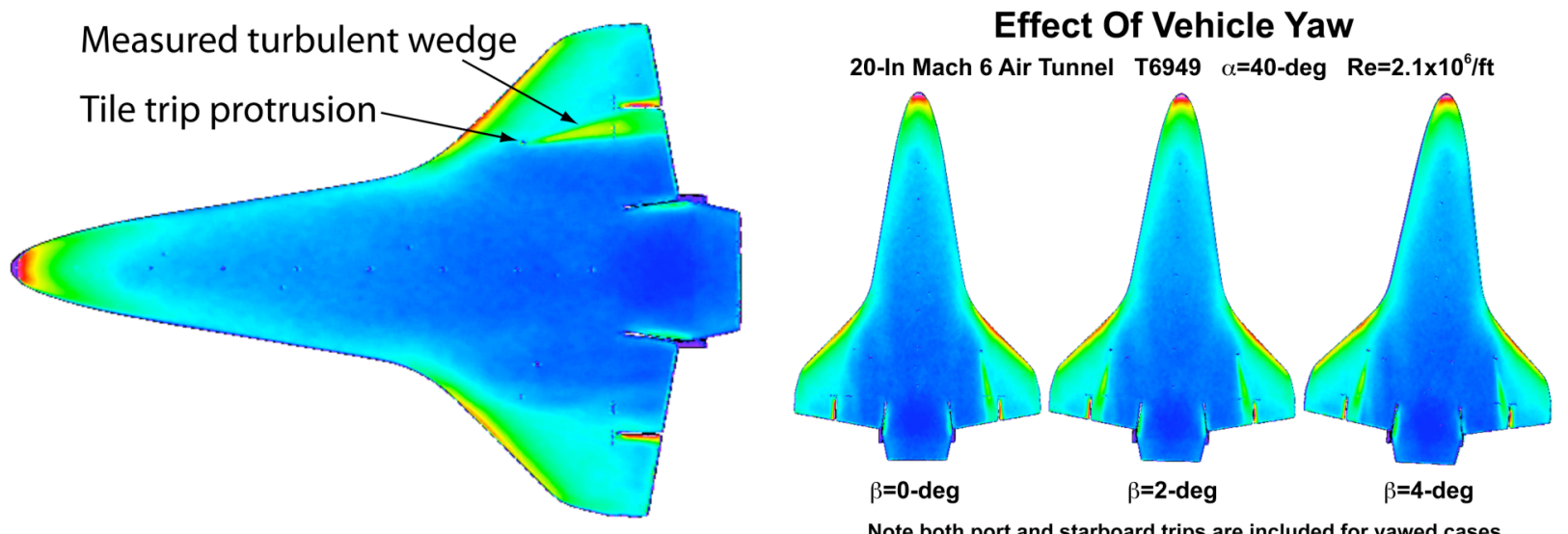

Figure 9. Wind tunnel result showing location of Figure 10. Wind tunnel results showing location of Shuttle BLT FE trip and measured turbulent wedge.

8

American Institute of Aeronautics and Astronautics 092407 
for the two yaw cases shown, trips were placed on both the port and starboard wings (and only placed on the starboard side for the 0 -deg yaw case). By closely comparing images, one can see that 4 deg of yaw was required to see a significant shift in the wedge patterns, which far exceeds what the Shuttle would experience under nominal conditions. Similarly, large angle of attack changes were required to see any appreciable shift in the location of the wedges.

\section{HYTHIRM Imaging}

As detailed in Ref. 9, the HYTHIRM project has multiple tasks, such as establishing an understanding of the capabilities and experience of national assets to obtain IR imagery, developing simulation and radiance modeling tools, and validation of the use of those tools and assets, that in the end are intended to allow recommendations of the best observation strategy for imaging the Shuttle during reentry in support of the Shuttle BLT FE. However, a recognized long-term goal of the HYTHIRM project is also to seek other opportunities to obtain global IR aerothermal data as possible support for other flight projects. An obvious near-term candidate for this is the HyBoLT experiment that is intended to fly this summer. As the first step in building an advocacy package for imaging of HyBoLT, the visual simulation capability of the team was applied knowing the geometry of the vehicle (CAD file) and the planned trajectory. Shown in Fig. 11 are two simulated images of HyBoLT based on an airborne platform located five miles from the ground track. The wind tunnel results previously discussed were mapped to CAD geometry as part of the simulation of the optical performance of the IR system. On the left of the figure is the visual result for Side A at the Mach number of interest for the natural transition experiment, indicating that approximately each camera pixel would represents 0.6 -in, which is sufficient to show such details as the flow structure around the two boundary layer probes at the aft end of Side A. On the right side is the visual result for Side B at the Mach number where the trips were designed for BLT onset, indicating roughly 1.3-in per pixel resolution. While the resulting Mach 7 simulated image is smaller, the three turbulent wedges can still be identified, suggesting adequate resolution. This simulation represents just the optical performance of a system at its best with no blurring due to atmospheric or vibration effects. Further, the images in Fig. 11 do not actually simulate the expected irradiance from the vehicle, as a radiance model of a copper body has not yet been developed. Currently there are no plans to image the HyBoLT launch, but from a technical perspective it does appear promising based strictly on surface resolution. Also, a recording of the flight could prove invaluable for reconstructing vehicle performance and health.

The identification and inventory of relevant optical imaging assets and the development, maturation, and validation of simulation and modeling tools for assessment and mission planning purposes were to lead to the best candidates and strategies for successful acquisition of quantitative global surface temperature data on the Shuttle. This process, essentially a trade study, has turned out to be harder than first thought due to the significant number of variables that are beyond the control of the HYTHIRM project. For instance, the ground track for any given mission may not be certain until the final minutes, with mission extensions and weather problems being the primary concerns. The best strategy for the planned nominal end-of-mission may not be ideal for one-revolution or even one-day wave offs. And if multiple weather delays occur, the landing site could even be changed from the east coast to the west coast. Figure 12 shows the variability of many of the recent flights to ISS, with some landing in $\mathrm{KSC}$ and some at Edwards AFB. One observation from this figure is the low probability of having an entry that is mostly over land (here only 3 of 20), which then favors the flexibility and range of the airborne systems. But even airborne systems cannot protect for a diversion to the west coast. The only way to do that is to utilize multiple systems spread out to cover all contingencies, which will drive up the cost of the project.

At this point in the planning process for support to the Shuttle BLT FE, flexibility appears to be the best strategy. A mixture (or quiver) of systems available to use at a moments notice allows the most flexibility to account for the last minute issues that crop up along the way to entry. A clear understanding of strengths and weaknesses
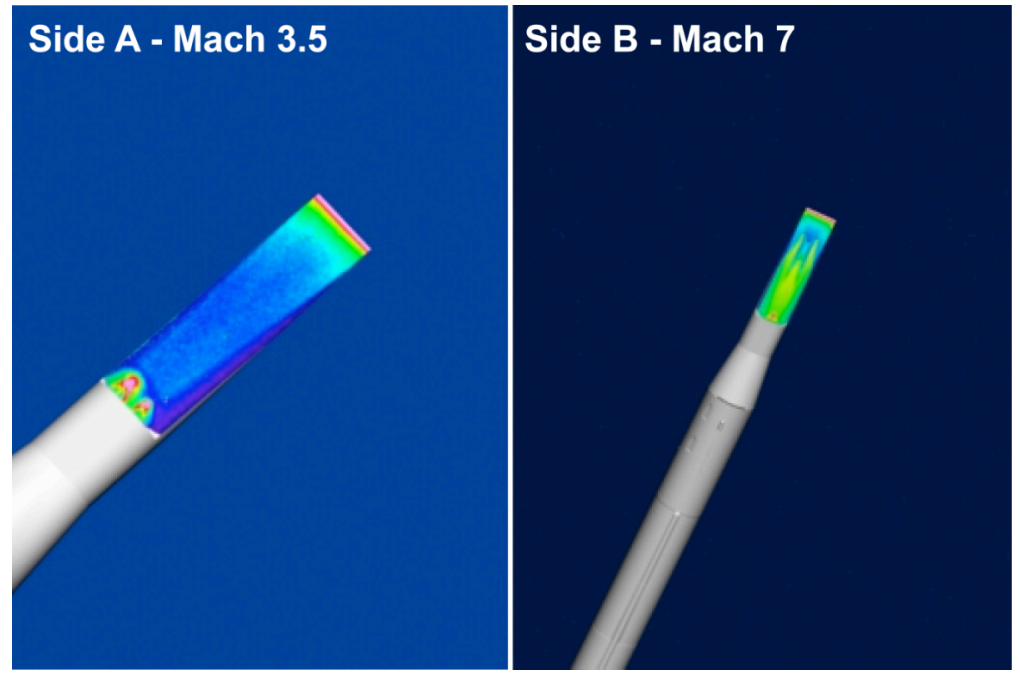

Figure 11. Optical simulation of the HyBoLT launch using an airborne system 5 miles from ground track.

9

American Institute of Aeronautics and Astronautics 092407 
of airborne and land-based systems is essential to successful mission support. For aircraft, the strengths are they are highly mobile, operate above a predominance of the weather, can be deployed over large bodies of water, and are typically able to handle one-orbit wave-offs. Their primary weaknesses include operational expense, and occasional difficulty with schedule conflicts due to requirements from other programs. For land-based systems, the strengths include much lower operational expense, high quality and large aperture optics for excellent performance, they can be made to be relatively mobile (to support one day wave-offs), and are typically not as susceptible to schedule conflicts. Weaknesses include increased vulnerability to weather (below clouds and moisture), not able to support one-orbit wave-offs, and require some logistical considerations (access roads and power). Both airborne and land-based systems have to consider seeking state department

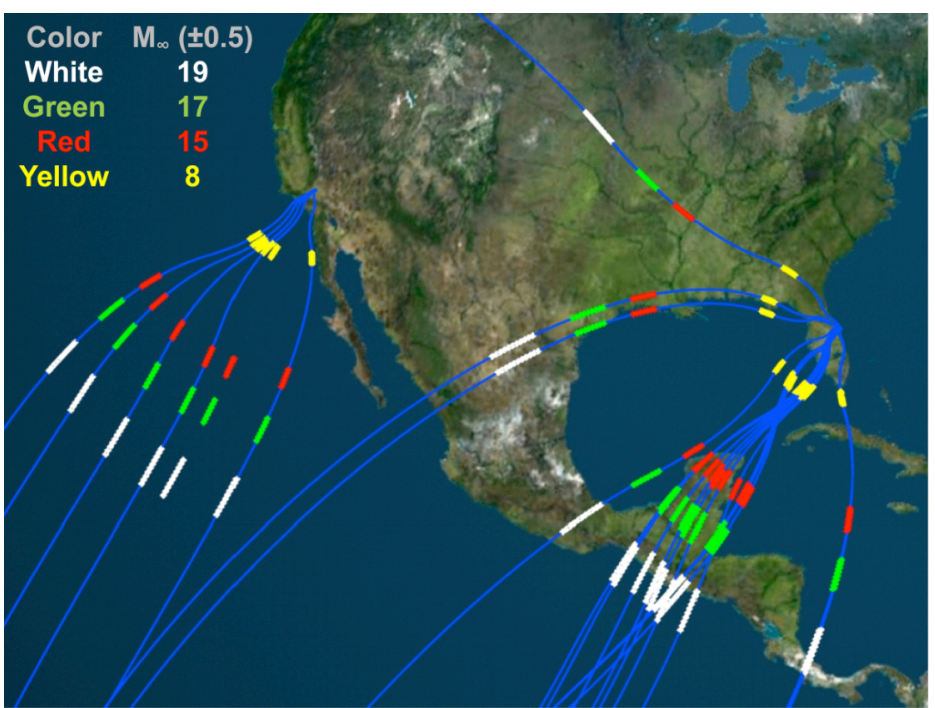

Figure 12. Recent Shuttle entry trajectories for return from International Space Station. permission for international deployments. Reference 10 provides additional details about the trade study and recommendations. A likely scenario for an imaging campaign would include one or two aircraft (depending on availability) and several land based systems (depending on how much of the nominal ground track will be over land).

The HYTHIRM team has developed a radiance model for the Shuttle, see Ref. 13. Using a nominal Shuttle trajectory and the known performance characteristics of the many imaging assets available, simulated IR images can then be produced, allowing selection of the best candidates for the mission, or to make recommendations for system improvements. Shown in Fig. 13 is a simulated IR image for one of the systems from Cast Glance, a mid-wave IR camera, indicating adequate resolution to observe the turbulent wedge on the wing emanating from the BLT FE trip site (note these simulations erroneously have the trip site on the starboard side). If instead a near IR system on WAVE is used, see Fig. 14, better optical resolution is obtained, however the temperature resolution is now less than optimum as parts of the underside of the Orbiter are off-scale low (black regions). These radiance simulations can be used to make recommendations on the dynamic range and exposure time settings for each system in order to maximize the quality of the resulting images. Both of these simulations suggest resolution better than the stated goal of $18 \mathrm{in} / \mathrm{pixel}$ (see Ref. 10), even with the assumed 2-pixel Gaussian point spread function that was used to account for blurring from typical optical diffraction limits and atmospheric effects. Note that an advantage of the WAVE aircraft is that it flies at a higher altitude, and thus closer to the Shuttle overhead and it currently has longer focal length optics available. However, a current disadvantage of WAVE is an optical imaging system that, as of yet, has not demonstrated the ability to lock-on and track an Orbiter without significant sensor motion (or jitter). WAVE

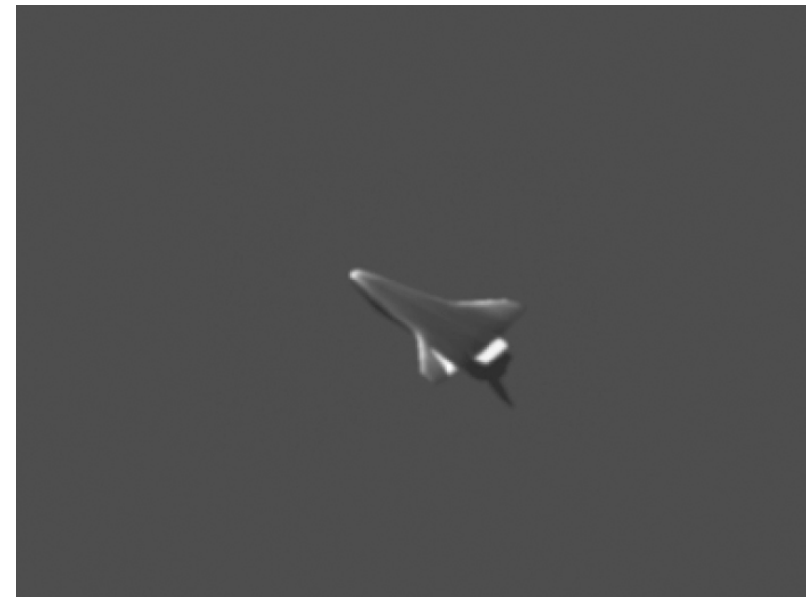

Figure 13. Simulated mid-wave IR intensity image based on Cast Glance specifications.

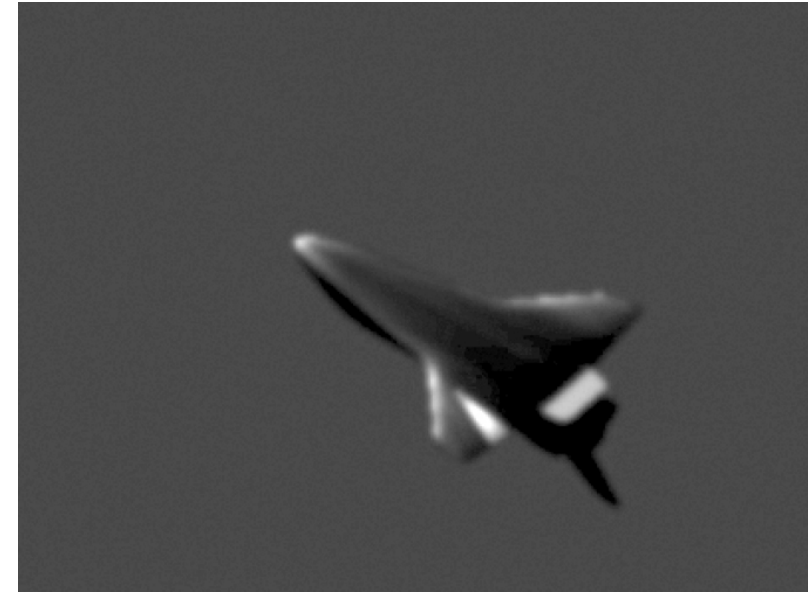

Figure 14. Simulated near IR intensity image based on WAVE specifications. 
personnel are presently working to alleviate these concerns. Both of these simulations are for a Mach 15 trip (the first BLT FE), with the aircraft approximately 10 miles from the ground track. For typical trajectories, as shown in Fig. 12, it is likely that the aircraft would be stationed near the Yucatan peninsula, where a majority of the red portions of the ground tracks are shown.

\section{Summary}

HYTHIRM is primarily focused on near term support to the Shuttle program with the development of an infrared imaging capability of sufficient spatial and temporal resolution to augment the existing on-board instrumentation. Significant progress has been made with the identification and inventory of relevant existing optical imaging assets and the development, maturation, and validation of simulation and modeling tools for assessment and mission planning purposes. At present, work is progressing on optimizing strategies and assets for successful acquisition of quantitative global surface temperature data on the Shuttle. Long-term goals of the HYTHIRM project include developing a plan for global IR imaging support to other flight projects as well. Two near-term NASA-sponsored boundary layer transition flight experiments, HyBoLT and the Shuttle BLT Flight Experiment, are discussed, which could benefit by infrared measurements. Those two flight projects have been proposed for reducing uncertainties with the extrapolation of wind tunnel derived BLT correlations for flight application. Thus, the criticality of obtaining high quality BLT flight data is reviewed along with the impact it would provide to the Shuttle program damage assessment process. Risk mitigation, in terms of quantifying the transition process and resulting turbulent wedge locations, was conducted through two separate wind tunnel efforts. From the HyBoLT test, an observation was that the outboard trips were swept further outboard than expected. From the Shuttle BLT FE test, the turbulent wedge at wind tunnel conditions was shown to be more inboard and smaller than predicted at flight conditions, the significance of which is still being assessed. The simulation of the optical capability of a typical airborne system imaging HyBoLT revealed that resolution on the order of 1-in per pixel was achievable; however there has been no request by the program, as of yet, to support the launch. Progress is being made towards finalizing an imaging strategy in support of the Shuttle BLT FE, with multiple landbased and airborne assets likely to be proposed for the effort.

\section{References}

\footnotetext{
${ }^{1}$ Berry, S. A., Horvath, T. J., Greene, F. A., Kinder, G. R., and Wang, K.C., "Overview of Boundary Layer Transition Research in Support of Orbiter Return to Flight," AIAA-2006-2918, June 2006.

${ }^{2}$ Campbell, C., Anderson, B., Bourland, G., Bouslog, S., Cassady, A., Horvath, T., Berry, S., Gnoffo, P., Wood, B., Reuther, J., Driver, D., Chao, D., and Picetti, D., "Orbiter Return To Flight Entry Aeroheating," AIAA-2006-2917, June 2006.

${ }^{3}$ Horvath, T., Berry, S., Merski, N, Berger, K., Liechty, D., Buck, G., and Schneider, S., "Shuttle Damage/Repair From the Perspective of Hypersonic Boundary Layer Transition - Experimental Results," AIAA-2006-2918, June 2006.

${ }^{4}$ Greene, F. A., and Hamilton, H., "Development of a Boundary Layer Properties Interpolation Tool in Support of Orbiter Return-To-Flight," AIAA-2006-2920, June 2006.

${ }^{5}$ McGinley, C., Berry, S. A., Kinder, G. R., Barnwell, M., Wang, K. C., and Kirk, B. S., "Review of Orbiter Flight Boundary Layer Transition Data," AIAA-2006-2921, June 2006.

${ }^{6}$ Berry, S. A., Horvath, T. J., Cassady, A. M., Kirk, B. S., Wang, K.C., and Hyatt, A. J., "Boundary Layer Transition Results From STS-114," AIAA-2006-2922, June 2006.

${ }^{7}$ Campbell, C. H., Garske, M. T., Kinder, J., and Berry, S. A., “Orbiter Entry Boundary Layer Flight Testing,” AIAA-20080635, Jan., 2008.

${ }^{8}$ Berry, S., Chen, F., Wilder, M., and Reda, D., "Boundary Layer Transition Experiments in Support of the Hypersonics Program," AIAA Paper 2007-4266, June. 2007.

${ }^{9}$ Horvath, T., Berry, S., Splinter, S., Daryabeigi, K., Wood, W., Schwartz, R. and Ross, M., "Assessment and Mission Planning Capability For Quantitative Aerothermodynamic Flight Measurements Using Remote Imaging,” AIAA 2008-4022, June 2008.

${ }^{10}$ Schwartz, R., Ross, M., Baize, R., Horvath, T., Berry, S., and Krasa, P., "A System Trade Study of Remote Infrared Imaging for Space Shuttle Re-entry," AIAA-2008-4023, June 2008.

11 Splinter, S., Daryabeigi, K., Mercer, C.D., Ghanbari, C., Tietjen, A., Schwartz, R., "Solar Tower Experiments for Radiometric Calibration and Validation of Infrared Imaging Assets and Analysis Tools for Entry Aero-Heating Measurements," AIAA-2008-4025, June 2008.

${ }^{12}$ Horvath, T., Berry, S., Alter, S., Blanchard, R., Schwartz, R., Ross, M., and Tack, S., "Shuttle Entry Imaging Using Infrared Thermography," AIAA-2007-4267, June 2007.

${ }^{13}$ Ross, M., Werner, M., Mazuk, S., Blanchard, R., Horvath, T., Berry, S., Wood, W., and Schwartz, R., "Infrared Imagery of the Space Shuttle at Hypersonic Entry Conditions," AIAA-2008-0636, Jan. 2008.

${ }^{14}$ Campbell, C.H., Driver, D.M., Alter, S.J., Fasanella, E.L., Wood, W.A., and Stone, J.S., "Orbiter Gap Filler Bending Model for Re-entry," AIAA Paper 2007-413, Jan. 2007.

${ }^{15}$ Miller, C. G., "Langley Hypersonic Aerodynamic/ Aerothermodynamic Testing Capabilities - Present and Future," AIAA Paper 90-1376, June 1990.
} 